\title{
COVID-19 Testing and Incidence Among Uninsured and Insured Individuals in 2020: a National Study
}

J Gen Intern Med 37(5):1344-7

DOI: $10.1007 / \mathrm{s} 11606-022-07429-0$

(C) The Author(s) under exclusive licence to Society of General Internal Medicine 2022

\section{BACKGROUND}

COVID-19 testing allows case identification, initiation of isolation, contact tracing, and prompt treatment. However, lack of health insurance may obstruct timely testing.

\section{METHODS}

We analyzed 11,824 adults $18-64$ in the 2020 National Health Interview Survey (quarters 3-4).

The primary exposure was any versus no insurance coverage at the time of interview ( $n=24$ excluded because of missing insurance status). We examined four outcomes: having (A) had $\geq 1$ COVID-19 test (hereinafter "tested"); (B) a positive test among those tested ("test-positivity"); (C) been told by a doctor or other health professional that you "had or likely had" COVID-19 ("clinician-reported diagnosis"); and (D) a positive result on any COVID-19 test among the full study population, i.e. not just among those tested ("COVID-19 incidence by test").

We examined the proportion of individuals with each outcome stratified by insurance in the full study population and among those with three common chronic diseases thought to increase risk for severe COVID-19: asthma, hypertension, and diabetes.

We performed multivariable logistic regressions (overall and among subgroups) examining the association between coverage status and each of our outcomes, adjusted for age, sex, region, urban/rural status, self-reported health, and income (excluding $n=7$ lacking data on a covariate).

We used Stata 16 procedures that account for the complex sampling methodology and weights that allow extrapolation nationally.

Received December 7, 2021

Accepted January 19, 2022

Published online February 9, 2022

\section{FINDINGS}

Table 1 provides the characteristics of $n=1295$ uninsured and $n=10,505$ insured adults in our sample. Relative to the insured, uninsured individuals were younger and had lower income; more were Hispanic (and fewer White non-Hispanic) and lived in Southern states. They had similar self-reported health and rates of asthma and diabetes, but less diagnosed hypertension.

Table 2 provides COVID-19 testing and incidence data. $32.8 \%$ of the insured were tested compared to $26.2 \%$ of the uninsured $(p<0.001)$; the adjusted difference was similar (6.6 percentage points [95\% CI $-9.8,-3.4]$ ). Among those tested, the positivity rate was almost double among uninsured $(21.7 \%)$ relative to insured $(11.1 \%)$ individuals (adjusted difference 9.4 percentage points [95\% CI 3.1, 15.6]).

Rates of clinician-reported COVID-19 diagnosis did not differ. Unadjusted incidence by test was higher among the uninsured $(5.4 \%)$ vs. the insured $(3.5 \%)$, although the difference was non-significant in the adjusted analysis $(p=0.07)$.

We observed mostly similar patterns among those with each medical condition, although some differences were nonsignificant.

\section{DISCUSSION}

In 2020, uninsured adults (including many with chronic diseases) were less likely than the insured to have been tested for COVID-19 despite having higher rates of positive test results.

The Families First Coronavirus Act (revised by the CARES Act) required insurers to cover COVID-19 testing without cost sharing, and provided public payment for testing uninsured individuals. However, it allowed providers not seeking public reimbursement to charge uninsured patients for testing. A secret shopper study found that $84 \%$ of urgent care centers charged uninsured individuals for COVID-19 testing. ${ }^{1}$ Media reports, ${ }^{2}$ moreover, have suggested that many individuals received large bills for testing despite the regulations. It seems likely that cost concerns deterred some uninsured individuals from seeking testing.

Our finding that the uninsured had a higher unadjusted incidence of positive COVID-19 tests, but not clinicianreported infections, probably reflects disparities in access to 
Table 1 Characteristics of Insured and Uninsured Adults 18-64, Q3-Q4 2020 National Health Interview Survey $(n=11,800)$

\begin{tabular}{|c|c|c|c|}
\hline & \multicolumn{3}{|c|}{ Health insurance status" } \\
\hline & \multirow{2}{*}{$\frac{\text { Insured }(n=10,505)}{\text { Weighted } \%}$} & \multirow{2}{*}{$\frac{\text { Uninsured }(n=1295)}{\text { Weighted } \%}$} & \multirow[t]{2}{*}{$p$ value } \\
\hline & & & \\
\hline $\begin{array}{l}\text { Age } \\
18-24(n=875) \\
25-34(n=2351) \\
35-44(n=2688) \\
45-54(n=2529) \\
55-64(n=3357)\end{array}$ & $\begin{array}{l}14.5 \\
21.6 \\
20.7 \\
20.6 \\
22.6\end{array}$ & $\begin{array}{l}15.0 \\
29.7 \\
23.4 \\
17.8 \\
14.1\end{array}$ & $<0.001$ \\
\hline $\begin{array}{l}\text { Sex } \\
\quad \text { Male }(n=5654) \\
\text { Female }(n=6145)\end{array}$ & $\begin{array}{l}48.8 \\
51.2\end{array}$ & $\begin{array}{l}51.0 \\
49.0\end{array}$ & 0.19 \\
\hline $\begin{array}{l}\text { Family income } \\
\$ 0 \text { to } \$ 34,999(n=2526) \\
\$ 35,000 \text { to } \$ 49,999(n=1353) \\
\$ 50,000 \text { to } \$ 74,999(n=2093) \\
\$ 75,000 \text { to } \$ 99,999(n=1568) \\
\$ 100,000 \text { or greater }(n=4260)\end{array}$ & $\begin{array}{l}18.2 \\
10.9 \\
18.0 \\
13.9 \\
39.1\end{array}$ & $\begin{array}{l}40.9 \\
19.1 \\
17.7 \\
10.6 \\
11.7\end{array}$ & $<0.001$ \\
\hline $\begin{array}{l}\text { Race/ethnicity } \\
\text { Hispanic }(n=1718) \\
\text { Non-Hispanic White }(n=7757) \\
\text { Non-Hispanic Black }(n=1255) \\
\text { Non-Hispanic other }(n=1070)^{\S}\end{array}$ & $\begin{array}{l}14.9 \\
63.4 \\
12.3 \\
9.4\end{array}$ & $\begin{array}{l}43.7 \\
36.1 \\
12.9 \\
7.3\end{array}$ & $<0.001$ \\
\hline $\begin{array}{l}\text { Household region } \\
\text { Northeast }(n=2024) \\
\text { Midwest }(n=2786) \\
\text { South }(n=3948) \\
\text { West }(n=3042)\end{array}$ & $\begin{array}{l}18.4 \\
22.1 \\
34.8 \\
24.6\end{array}$ & $\begin{array}{l}8.6 \\
15.7 \\
54.1 \\
21.6\end{array}$ & $<0.001$ \\
\hline $\begin{array}{l}\text { County urbanity/rurality } \\
\text { Large central metro }(n=3720) \\
\text { Large fringe metro }(n=2727) \\
\text { Medium and small metro }(n=3758) \\
\text { Nonmetropolitan }(n=1595)\end{array}$ & $\begin{array}{l}31.6 \\
25.7 \\
30.4 \\
12.2\end{array}$ & $\begin{array}{l}32.4 \\
21.4 \\
31.4 \\
14.8\end{array}$ & 0.13 \\
\hline $\begin{array}{l}\text { Self-reported health status } \\
\text { Good or better }(n=10,442) \\
\text { Fair or poor }(n=1352)\end{array}$ & $\begin{array}{l}88.7 \\
11.3\end{array}$ & $\begin{array}{l}88.7 \\
11.3\end{array}$ & 0.97 \\
\hline $\begin{array}{l}\text { Asthma (current) })^{\mathrm{T}} \\
\text { No }(n=10,768) \\
\text { Yes }(n=1002)\end{array}$ & $\begin{array}{l}91.2 \\
8.8\end{array}$ & $\begin{array}{l}92.2 \\
7.8\end{array}$ & 0.37 \\
\hline $\begin{array}{l}\text { Hypertension diagnosis } \\
\text { No }(n=8791) \\
\text { Yes }(n=2993)\end{array}$ & $\begin{array}{l}76.0 \\
24.0\end{array}$ & $\begin{array}{l}81.2 \\
18.8\end{array}$ & $<0.01$ \\
\hline $\begin{array}{l}\text { Diabetes (ever) } \\
\text { No }(n=10,998) \\
\text { Yes }(n=792)\end{array}$ & $\begin{array}{l}93.8 \\
6.2\end{array}$ & $\begin{array}{l}94.7 \\
5.3\end{array}$ & 0.32 \\
\hline
\end{tabular}

Number with missing data: $\operatorname{sex}(n=1)$; self-rated health $(n=6)$; current asthma $(n=30)$; hypertension $(n=16) ;$ diabetes $(n=10)$

"Individuals are classified as uninsured in the NHIS if they lack private health insurance, Medicare, Medicaid, CHIP, state-sponsored health plan, other government health plan, or military health plan when interviewed, and insured if they have one of these forms of coverage (note that this definition excludes those with only Indian Health Service as insured)

Includes singly imputed NHIS values

${ }^{\S}$ Other includes non-Hispanic Asian, non-Hispanic American Indian, non-Hispanic American Indian and other, and "other single and multiple races" "2013 NCHS Urban-Rural Classification Scheme for Counties

"Assessed with the question: "Have you EVER been told by a doctor or other health professional that you had asthma?," followed by "Do you still have asthma?"

*Assessed with the question: "Have you EVER been told by a doctor or other health professional that you had... Hypertension, also called high blood pressure?"

${ }^{H}$ Assessed with the question “... a doctor or other health professional EVER told you that you had diabetes?”, excluding gestational diabetes and prediabetes

clinicians - findings consonant with a trend toward a larger increase in all-cause mortality among the uninsured relative to the insured in $2020 .^{3}$

Our nationwide analysis extends previous reports of language-, ${ }^{4}$ race/ethnicity-, ${ }^{5}$ and socioeconomic-based ${ }^{6}$ testing disparities in several locales. A limitation of our study is that test results were self-reported. Underdiagnosis of COVID-19 was likely greater among the uninsured, as reflected in higher test positivity. Although we controlled for multiple confounders, observational studies cannot prove causality. Representativeness of NHIS respondents is a potential concern; however, the proportion of our sample with a positive COVID-19 test is similar to the proportion estimated from publicly available CDC case microdata (authors' unpublished analysis). 
Table 2 COVID-19 Testing and Incidence According to Insurance Status, Adults Ages 18-64, $2020(n=11,800)$

\begin{tabular}{|c|c|c|c|c|c|c|c|c|c|}
\hline & \multicolumn{4}{|c|}{ Unadjusted $^{\|}$} & \multicolumn{5}{|c|}{ Adjusted $^{\pi}$} \\
\hline & $\begin{array}{l}n \text { for } \\
\text { analysis }\end{array}$ & $\begin{array}{l}\text { Insured } \\
(\%)\end{array}$ & $\begin{array}{l}\text { Uninsured } \\
(\%)\end{array}$ & $p$ value & $\begin{array}{l}n \text { for } \\
\text { analysis }\end{array}$ & $\begin{array}{l}\text { Percentage } \\
\text { point } \\
\text { difference }\end{array}$ & $\begin{array}{l}95 \% \\
\text { confid } \\
\text { interv }\end{array}$ & & $p$ value \\
\hline \multicolumn{10}{|l|}{ Overall population } \\
\hline Tested & 11,746 & 32.8 & 26.2 & $<0.001$ & 11,740 & -6.6 & -9.8 & -3.4 & $<0.001$ \\
\hline Test positivity ${ }^{\dagger}$ & 3700 & 11.1 & 21.7 & $<0.001$ & 3699 & 9.4 & 3.1 & 15.6 & 0.003 \\
\hline $\begin{array}{l}\text { Clinician-reported } \\
\text { diagnosis }\end{array}$ & 11,755 & 4.6 & 6.0 & 0.10 & 11,748 & 1.1 & -0.7 & 2.9 & 0.24 \\
\hline $\begin{array}{l}\text { COVID-19 incidence } \\
\text { by test }\end{array}$ & 11,800 & 3.5 & 5.4 & 0.010 & 11,793 & 1.7 & -0.1 & 3.4 & 0.067 \\
\hline \multicolumn{10}{|l|}{ Asthma } \\
\hline Tested & 999 & 40.0 & 26.9 & 0.058 & 999 & -15.4 & -27.0 & -3.8 & 0.009 \\
\hline Test positivity & 391 & 9.7 & 24.9 & 0.052 & 391 & 20.6 & -0.3 & 41.5 & 0.053 \\
\hline $\begin{array}{l}\text { Clinician-reported } \\
\text { diagnosis }\end{array}$ & 1000 & 6.1 & 8.8 & 0.42 & 1000 & 3.4 & -3.9 & 10.7 & 0.36 \\
\hline $\begin{array}{l}\text { COVID-19 incidence } \\
\text { by test }\end{array}$ & 1002 & 3.7 & 6.7 & 0.27 & 1002 & 3.3 & -3.5 & 10.1 & 0.34 \\
\hline \multicolumn{10}{|l|}{ Hypertension } \\
\hline Tested & 2977 & 33.3 & 33.2 & 0.97 & 2976 & -1.0 & -9.0 & 7.1 & 0.81 \\
\hline Test positivity & 960 & 8.4 & 31.5 & $<0.001$ & 960 & 21.6 & 8.0 & 35.1 & 0.002 \\
\hline $\begin{array}{l}\text { Clinician-reported } \\
\text { diagnosis }\end{array}$ & 2981 & 4.0 & 10.5 & 0.002 & 2980 & 4.7 & -0.2 & 9.6 & 0.059 \\
\hline $\begin{array}{l}\text { COVID-19 incidence } \\
\text { by test }\end{array}$ & 2993 & 2.7 & 10.0 & $<0.001$ & 2992 & 6.2 & 1.4 & 11.0 & 0.012 \\
\hline \multicolumn{10}{|l|}{ Diabetes } \\
\hline Tested & 786 & 36.6 & 30.6 & 0.49 & 786 & -5.0 & -20.3 & 10.2 & 0.52 \\
\hline Test positivity & 276 & 10.8 & 45.4 & 0.007 & 267 & 29.8 & 8.3 & 51.3 & 0.007 \\
\hline $\begin{array}{l}\text { Clinician-reported } \\
\text { diagnosis }\end{array}$ & 788 & 4.7 & 13.9 & 0.064 & 759 & 8.1 & -1.5 & 17.7 & 0.10 \\
\hline $\begin{array}{l}\text { COVID-19 incidence } \\
\text { by test }\end{array}$ & 792 & 3.8 & 13.9 & 0.032 & 763 & 7.6 & -1.1 & 16.4 & 0.088 \\
\hline
\end{tabular}

" "Tested" assessed with the question: "Have you ever been tested for coronavirus or COVID-19?" Those with "refused," "not ascertained," or "don't know" answers were treated as missing and excluded from the analysis $(n=54)$

"Test positivity" was assessed only among those who indicated having been tested ( $n=3803)$, with the question: "Did the test find that you had coronavirus or COVID-19?" Those with "did not receive results" or "don't know" responses were treated as missing. Among those who reported testing, $n=103$ had missing data on results

* "Clinician-reported diagnosis" was assessed with the question: "Has a doctor or other health professional ever told you that you had or likely had coronavirus or COVID-19?" Those with "refused," "not ascertained," or "don't know" answers were treated as missing and excluded from the analysis $(n=45)$

\& "COVID-19 Incidence by test" was assessed with the same question as used for "test positivity," but the entire study population was used as the denominator, such that those not tested or with unknown testing status or results were treated as having a negative result. Hence, there was no missing data for the study population of $n=11,800$

"l value from univariate logistic regression

${ }^{\top}$ We used Stata's margins command to calculate adjusted percentage point differences (and confidence intervals and p values) from our multivariable logistical regressions. All models adjusted for age (18-24, 25-34, 35-44, 45-54, and 55-64 years); gender (male, female); region (Northeast, Midwest, South, West); urban/rural county (large central metro, large fringe metro, medium and small metro, nonmetropolitan); self-reported health status (fair or poor vs. good or better); and family income (\$0-\$34,999, \$35,000-\$49,999, \$50,000-\$74,999, \$75,000-\$99,999, $\$ 100,000+)$. $n=7$ with missing data on one or more covariates were excluded from analyses. Family income variable was singly imputed by the NHIS

The mismatch we observed between testing and COVID-19 risk has important implications. New antiviral medications require prompt diagnosis (and initiation) after symptom onset to be effective; disparities in testing access could impede the equitable use (and efficacy) of such agents. Moreover, the Biden administration's requirement that insurers cover home rapid antigen tests will not aid the uninsured, potentially widening testing disparities.

Further coverage expansion is needed to achieve more equitable and effective COVID-19 care.
Adam Gaffney, MD, $M P H^{1,2}$

Steffie Woolhandler, MD MPH ${ }^{1,2,3}$

David U. Himmelstein, $M D^{1,2,3}$

${ }^{1}$ Cambridge Health Alliance,

Cambridge, MA, USA

${ }^{2}$ Harvard Medical School,

Boston, MA, USA

${ }^{3}$ City University of New York at Hunter College,

New York, NY, USA 
Corresponding Author: Adam Gaffney, MD, MPH; Harvard Medical School, Boston, MA, USA (e-mail: agaffney@challiance.org).

\section{Declarations:}

Conflict of Interest: The authors report no financial conflicts of interest. Adam Gaffney, David Himmelstein, and Steffie Woolhandler are, or have served as, leaders of Physicians for a National Health Program (PNHP), a non-profit organization that favors coverage expansion through a single-payer program; however, none of them received any compensation from that group, although some of Dr. Gaffney's travel on behalf of the organization has been reimbursed by it. Adam Gaffney's spouse is an employee of Treatment Action Group (TAG), a non-profit research and policy think tank focused on HIV, TB, and hepatitis $C$ treatment.

\section{REFERENCES}

1. Yousman LC, Khunte A, Hsiang W, Jain S, Forman H, Wiznia D. Urgent care center wait times increase for COVID-19 results in August 2020, with rapid testing availability limited. BMC Health Serv Res. 2021;21(1):318.

2. Kliff S. Two Friends in Texas Were Tested for Coronavirus. One Bill Was \$199. The Other? \$6,408. The New York Times [Internet]. 202029 [cited 2021 Nov 1]; Available from: https://www.nytimes.com/2020/06/29/ upshot/coronavirus-tests-unpredictable-prices.html

3. Miller S, Wherry LR, Mazumder B. Estimated Mortality Increases During The COVID-19 Pandemic By Socioeconomic Status, Race, And Ethnicity. Health Aff (Millwood). 2021;40(8):1252-60.

4. Kim HN, Lan KF, Nkyekyer E, Neme S, Pierre-Louis M, Chew L, et al. Assessment of Disparities in COVID-19 Testing and Infection Across Language Groups in Seattle, Washington. JAMA Netw Open. 2020;3(9):e2021213.

5. Grigsby-Toussaint DS, Shin JC, Jones A. Disparities in the distribution of COVID-19 testing sites in black and Latino areas in New York City. Prev Med. 2021;147:106463.

6. Dryden-Peterson $\mathbf{S}$, Velásquez GE, Stopka TJ, Davey $\mathbf{S}$, Lockman $\mathbf{S}$, Ojikutu BO. Disparities in SARS-CoV-2 Testing in Massachusetts During the COVID-19 Pandemic. JAMA Netw Open. 2021;4(2):e2037067.

Publisher's Note Springer Nature remains neutral with regard to jurisdictional claims in published maps and institutional affiliations. 